\title{
XRF 060428B: Observational evidence for a strongly lensed burst
}

\author{
D. A. Perley*, J. S. Bloom*, N. R. Butler*, W. Li* and H.-W. Chen ${ }^{\dagger}$ \\ *Department of Astronomy, 601 Campbell Hall, University of California, Berkeley, CA 94720-3411 \\ ${ }^{\dagger}$ Department of Astronomy and Astrophysics, University of Chicago, 5640 S. Ellis Ave, Chicago, IL \\ 60637
}

\begin{abstract}
Long-duration GRBs and X-ray aashes (XRFs; softer-spectrum brethren of long GRBs) are thought to occur following the core-collapse of massive stars. We report here on observations of a recent X-ray wash (XRF 060428B) that occurred 2.6" in projection from the center of a massive red galaxy at redshift $z=0.348$, well within its detectable light. While initial probabilistic arguments suggested a physical connection, deep Keck imaging reveals a compact blue source at the burst position, likely a higher-redshift host galaxy. Since the observed offset is approximately equal to the Einstein radius of the foreground elliptical, we suggest that XRF 060428B may have been strongly gravitationally lensed, allowing us to detect an underluminous burst at high-z. This would naturally explain the otherwise coincidental proximity to a nearby galaxy.
\end{abstract}

Keywords: gamma rays: bursts, gamma-ray bursts: individual: 060428B, gravitational lensing

PACS: $98.70 . \mathrm{Rz}, 98.62 . \mathrm{Sb}$

\section{XRF 060428B: A FAINT SWIFT X-RAY FLASH}

The Swift satellite [1] triggered on the burst 060428B on 2006 April 28 08:54:38 UTC [2]. We analyzed the public Swift data following standard analysis procedures. The duration of the event is $T_{90}=57.42 \mathrm{sec}$ and the peak in the spectral energy distribution, well $f t$ by a Band model [3], is $E_{p}=26.2_{-19.3}^{+28.9} \mathrm{keV}(2 \sigma)$. The duration and spectral softness of the burst place 060428B in the category of an X-Ray Flash (XRF) [4, 5].

Follow-up observations led to the identi£cation of a faint $(V \sim 19)$ optical afterglow consistent with the X-ray afterglow position [6]. A comparison of our early-time imaging of the afterglow with later-time imaging after the source faded (Figure 1) allows us to precisely locate this XRF $2.52^{\prime \prime}$ west and $0.05^{\prime \prime}$ north of a red source (G0) seen in the Sloan Digital Sky Survey [7].

\section{AN OLD GALAXY NEAR THE BURST POSITION}

G0 is a featureless, red elliptical galaxy. The projected location of the XRF falls in the outskirts of the galaxy, within the 80th percentile of the detected light.

We performed spectroscopic follow-up of this galaxy and several others in the feld with Keck I-LRIS on the nights of May 29 and July 27 (UT). The resulting spectra (Figure 2) show the galaxy to be clearly dominated by an old stellar population, with prominent absorption features and a pronounced $4000 \AA$ break. The galaxy is at a redshift of $z=0.348$, and has very little star formation. 


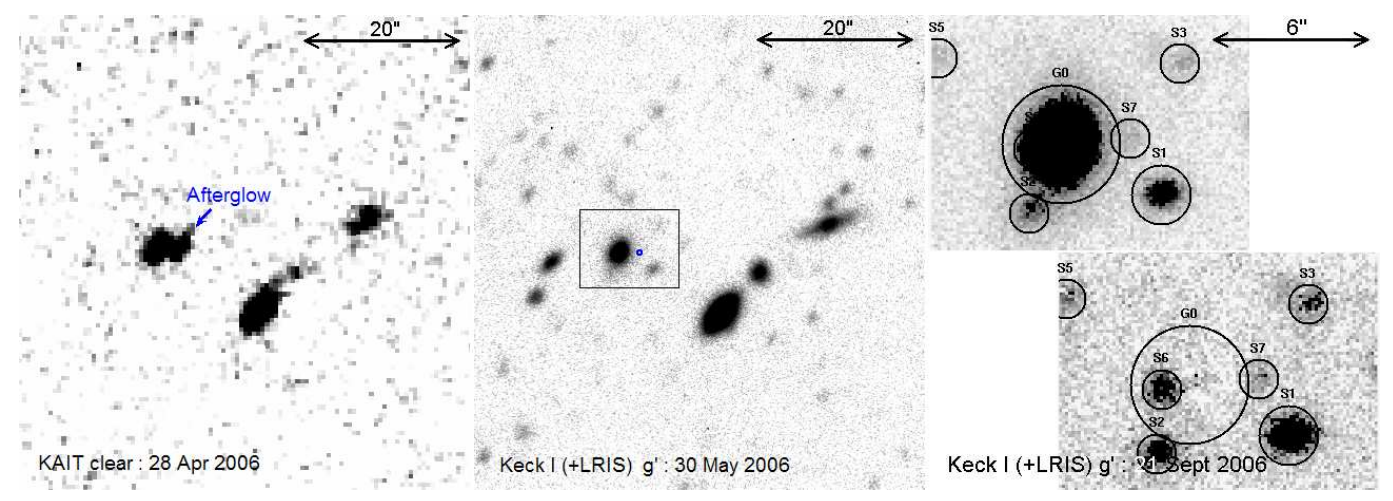

FIGURE 1. Imaging of the afterglow position and nearby galaxies, from KAIT and Keck. (Left Panel) The Katzmann Automated Imaging Telescope (KAIT) responded and began taking data 192 seconds after the burst trigger. The GRB was not detected in early exposures but is seen in longer-integration clear flters starting at $692 \mathrm{~s}$ after the trigger and ending at $2517 \mathrm{~s}$. It does not show evidence for fading during this interval. Shown here is a stacked exposure of 900 s with the optical afterglow identifed. (Middle Panel) Follow-up imaging was performed with Keck(+LRIS) on the nights of 30 May 2006 and 21 Sept 2006; $g$-band May imaging is shown. Astrometry (J2000) using the two images places the afterglow at $\alpha=15: 41: 25.628, \delta=+62: 01: 30.02$, offset $2.6^{\prime \prime}$ from the center of the putative host (at $\alpha=15: 41: 25.986$, $\delta=+62: 01: 29.97$ ). (Right Panel, Top) A zoomed in image of the burst location. (Right Panel, Bottom) A blue $(g-R=-0.35)$ source is visible at the afterglow position after subtracting the light of the foreground elliptical using the software package galft[8].

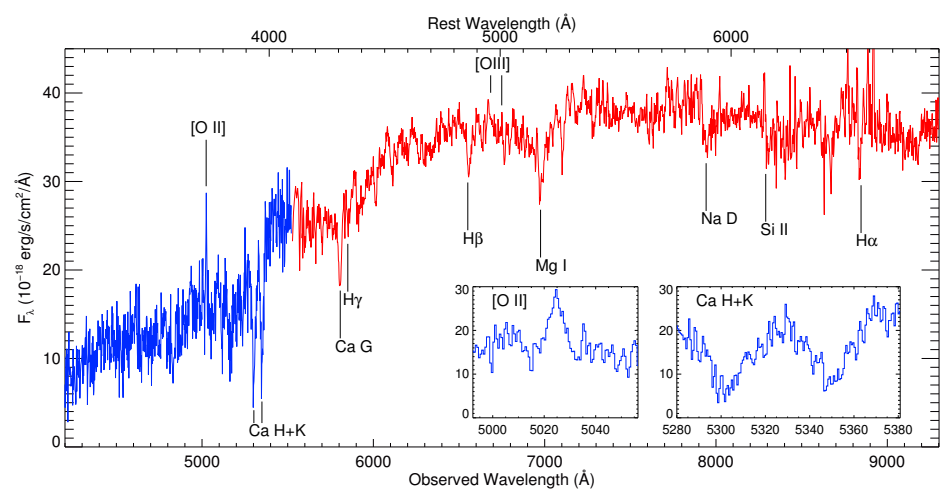

FIGURE 2. Spectrum from Keck I (+LRIS) of an old galaxy 2.6" from the location of XRF 060428B. We observed the source on the night of 26 July 2005 for 760s (blue) and 1800s (red). The galaxy displays signatures of an old stellar population with little star formation (weak emission lines) and prominent absorption (strong $\mathrm{Ca} \mathrm{II} \mathrm{H}+\mathrm{K}$ and Hydrogen Balmer series), in agreement with its overall red color and de Vaucouleurs light profle. From the weak but detected [OII] line we infer a star formation rate of $0.191 \pm 0.049 \mathrm{M}_{\odot} / \mathrm{yr}$. The dispersion velocity from $\mathrm{Mg} \mathrm{I}$ is $\sigma \approx 290 \mathrm{~km} / \mathrm{s}$, indicating a massive galaxy.

The characteristics of this galaxy are strikingly different from the hosts of previously known long-duration GRBs and XRFs, suggesting the possibility of a chance alignment. The probability of a single point on the sky chosen at random being within $2.6^{\prime \prime}$ of a galaxy with $r<20.0$ is very low; $P_{c h}=0.002$ [9]. However, there have been many previous GRBs and XRFs with optical afterglows but no spectroscopic redshift. The probability of at least one XRF from this sample appearing near a bright galaxy is still 
quite low at $P_{c h}=0.03$; for at least one such XRF or long-duration GRB it is relatively high at $P_{c h}=0.28$ (e.g. [10]).

\section{THE FAINT BACKGROUND HOST OF XRF060428B}

To search for a possible faint background host, we carried out deep imaging observations of the £eld with the Keck 10-meter telescope, and use the software package gal£t to subtract the light of the bright elliptical G0 and other nearby sources. Coincident with the afterglow position in our deep Keck imaging is a very faint blue source ("S7"), blended with the light of G0. Our a priori understanding of the origin of XRFs and long GRBs favors interpreting this galaxy as the true host of the XRF.

G0 is a massive galaxy. Fitting a convolved stellar template spectrum [11] to our LRIS spectrum for the Mg I triplet, we estimate a velocity dispersion of $290 \pm 60 \mathrm{~km} / \mathrm{s}$. Assuming a singular isothermal sphere for the galaxy's mass distribution and a nominal redshift range of the GRB of $1.1<\mathrm{z}<4.5$, the Einstein radius of G0 is $\theta_{E}=(3.8 \pm 1.4)^{\prime \prime}$. This puts the GRB location (at an offset of 2.6") within the region of strong lensing. Although very large magnifcations are ruled out by the lack of an obvious same-color host counterpart in our imaging, unless the source is at very low redshift $(z<0.5)$ it is likely to be at least $50 \%$ and probably signifcantly more.

A magnifcation factor on the order of a few would explain the apparent coincidence of the burst being aligned with a massive low-redshift galaxy that is not its host. The XRF was detected near the Swift threshold, and if the magnifcation factor is signi£cant, it is unlikely that Swift would have detected the burst without the presence of lensing. This is also consistent with the XRF nature of the event: XRFs are generally underluminous with respect to higher- $E_{\text {peak }}$ GRBs, and these soft, low-luminosity events are thought to be far more common than normal GRBs by at least an order of magnitude in terms of their absolute rates [12]. Ordinarily, similar events at high-z would fall below current detection thresholds; here, the presence of a foreground galaxy has provided a window on these events in the early universe. Though our probabilistic analysis suggests it is unlikely, it is also plausible that S7 is at relatively low redshift and unlensed; perhaps being cannibalized by the large elliptical. Further investigations are planned.

\section{REFERENCES}

1. N. Gehrels, G. Chincarini, P. Giommi, K. O. Mason, J. A. Nousek, A. A. Wells, N. E. White, S. D. Barthelmy, D. N. Burrows, L. R. Cominsky, K. C. Hurley, F. E. Marshall, P. Mészáros, P. W. A. Roming, L. Angelini, L. M. Barbier, T. Belloni, S. Campana, P. A. Caraveo, M. M. Chester, O. Citterio, T. L. Cline, M. S. Cropper, J. R. Cummings, A. J. Dean, E. D. Feigelson, E. E. Fenimore, D. A. Frail, A. S. Fruchter, G. P. Garmire, K. Gendreau, G. Ghisellini, J. Greiner, J. E. Hill, S. D. Hunsberger, H. A. Krimm, S. R. Kulkarni, P. Kumar, F. Lebrun, N. M. Lloyd-Ronning, C. B. Markwardt, B. J. Mattson, R. F. Mushotzky, J. P. Norris, J. Osborne, B. Paczynski, D. M. Palmer, H.-S. Park, A. M. Parsons, J. Paul, M. J. Rees, C. S. Reynolds, J. E. Rhoads, T. P. Sasseen, B. E. Schaefer, A. T. Short, A. P. Smale, I. A. Smith, L. Stella, G. Tagliaferri, T. Takahashi, M. Tashiro, L. K. Townsley, J. Tueller, M. J. L. Turner, M. Vietri, W. Voges, M. J. Ward, R. Willingale, F. M. Zerbi, and W. W. Zhang, ApJ 611, 1005-1020 (2004), astro-ph/ 0405233. 
2. S. Campana, S. D. Barthelmy, D. N. Burrows, J. R. Cummings, N. Gehrels, C. B. Markwardt, K. L. Page, D. M. Palmer, P. Romano, and D. E. V. Berk, GRB 060428B: Swift detection of a burst (2006), GCN Circular 5017.

3. D. Band, J. Matteson, L. Ford, B. Schaefer, D. Palmer, B. Teegarden, T. Cline, M. Briggs, W. Paciesas, G. Pendleton, G. Fishman, C. Kouveliotou, C. Meegan, R. Wilson, and P. Lestrade, ApJ 413, 281-292 (1993).

4. J. Heise, J. in't Zand, R. M. Kippen, and P. M. Woods, "X-Ray Flashes and X-Ray Rich Gamma Ray Bursts," in Gamma-ray Bursts in the Afterglow Era, edited by E. Costa, F. Frontera, and J. Hjorth, 2001, pp. 16-+.

5. R. M. Kippen, P. M. Woods, J. Heise, J. J. M. in’t Zand, M. S. Briggs, and R. D. Preece, "Spectral Characteristics of X-Ray Flashes Compared to Gamma-Ray Bursts," in AIP Conf. Proc. 662: Gamma-Ray Burst and Afterglow Astronomy 2001: A Workshop Celebrating the First Year of the HETE Mission, edited by G. R. Ricker, and R. K. Vanderspek, 2003, pp. 244-247.

6. P. A. Price, T. Minezaki, L. L. Cowie, Y. Kakazu, and Y. Yoshii, GRB 060428b: Optical afterglow candidate (2006), GCN Circular 5019.

7. R. J. Cool, D. J. Eisenstein, D. W. Hogg, M. R. Blanton, D. J. Schlegel, J. Brinkmann, D. Q. Lamb, D. P. Schneider, and D. E. V. Berk, GRB 060428B - SDSS Pre-Burst Observations (2006), GCN Circular 5037.

8. C. Y. Peng, L. C. Ho, C. D. Impey, and H.-W. Rix, AJ 124, 266-293 (2002), a stro-ph / 0204182.

9. N. Yasuda, M. Fukugita, V. K. Narayanan, R. H. Lupton, I. Strateva, M. A. Strauss,. \pm Ivezić, R. S. J. Kim, D. W. Hogg, D. H. Weinberg, K. Shimasaku, J. Loveday, J. Annis, N. A. Bahcall, M. Blanton, J. Brinkmann, R. J. Brunner, A. J. Connolly, I. Csabai, M. Doi, M. Hamabe, S.-I. Ichikawa, T. Ichikawa, D. E. Johnston, G. R. Knapp, P. Z. Kunszt, D. Q. Lamb, T. A. McKay, J. A. Munn, R. C. Nichol, S. Okamura, D. P. Schneider, G. P. Szokoly, M. S. Vogeley, M. Watanabe, and D. G. York, AJ 122, 1104-1124 (2001), astro-ph / 0105545 .

10. B. E. Cobb, C. D. Bailyn, P. G. van Dokkum, and P. Natarajan, ApJL 651, L85-L88 (2006), astro-ph/0608678.

11. J.-F. Le Borgne, G. Bruzual, R. Pelló, A. Lançon, B. Rocca-Volmerange, B. Sanahuja, D. Schaerer, C. Soubiran, and R. Vílchez-Gómez, A\&A 402, 433-442 (2003), astro-ph / 0302334.

12. A. M. Soderberg, S. R. Kulkarni, E. Nakar, E. Berger, P. B. Cameron, D. B. Fox, D. Frail, A. GalYam, R. Sari, S. B. Cenko, M. Kasliwal, R. A. Chevalier, T. Piran, P. A. Price, B. P. Schmidt, G. Pooley, D.-S. Moon, B. E. Penprase, E. Ofek, A. Rau, N. Gehrels, J. A. Nousek, D. N. Burrows, S. E. Persson, and P. J. McCarthy, Nature 442, 1014-1017 (2006), astro-ph/ 0604389. 